\title{
Evaluation of Outcome Prediction of Flow Diversion for Intracranial Aneurysms
}

\author{
(D) S. Hadad, (D)F. Mut, (D) R. Kadirvel, (D)Y.-H. Ding, (D) D. Kallmes, and (D).R. Cebral
} O-

\begin{abstract}
BACKGROUND AND PURPOSE: Identifying and predicting which aneurysms are likely to quickly occlude and which ones are likely to remain open following treatment with flow-diverting devices is important to develop optimal patient management strategies. The purpose of this study was to evaluate predictions based on computational fluid dynamics models using the elastase rabbit aneurysm model.
\end{abstract}

MATERIALS AND METHODS: A series of 13 aneurysms created in rabbits were treated with flow diverters, and outcomes were angiographically assessed at 8 weeks' follow-up. Computational fluid dynamics models were constructed from pretreatment 3D rotational angiograms and Doppler ultrasound flow velocity measurements. Postimplantation mean aneurysm inflow rate and flow velocity were used to prospectively predict aneurysm occlusion blinded to the actual outcomes. Specifically, if both variables were below their corresponding thresholds, fast occlusion was predicted, while if one of them was above the threshold, slow or incomplete occlusion was predicted.

RESULTS: Of the 13 aneurysms included, 8 were incompletely occluded 8 weeks after treatment, and 5 were completely occluded. A total of 10 computational fluid dynamics-based predictions agreed with the angiographic outcome, reaching $77 \%$ accuracy, $80 \%$ sensitivity, and $75 \%$ specificity. Posttreatment mean velocity alone was able to achieve the same predictive power as the combination of inflow rate and velocity.

CONCLUSIONS: Subject-specific computational fluid dynamics models of the hemodynamic conditions created immediately after implantation of flow-diverting devices in experimental aneurysms created in rabbits are capable of prospectively predicting, with a reasonable accuracy, which aneurysms will completely occlude and which ones will remain incompletely occluded.

ABBREVIATIONS: CFD = computational fluid dynamics; DUS = Doppler ultrasound; FD = flow-diverting (diverter); $Q=$ inflow rate; VEL $=$ velocity

$\mathbf{T}$ reatment of intracranial aneurysms with flow-diverting (FD) devices has been gaining popularity as a viable alternative to coiling or clipping, especially for complex aneurysms with wide necks. ${ }^{1}$ Many complex aneurysms have been successfully treated with an increasing variety of flow diverters. ${ }^{2}$ However, aneurysms are not immediately excluded from the

Received January 12, 2021; accepted after revision June 10.

From the Departments of Bioengineering and Mechanical Engineering (S.H., F.M., J.R.C.), George Mason University, Fairfax, Virginia; and Department of Interventional Neuroradiology (R.K., Y.-H.D., D.K.), Mayo Clinic, Rochester, Minnesota.

This work was supported by the National Institutes of Health, grant No. R01NS076491.

Please address correspondence to Sara Hadad, MSc, Bioengineering Department, Volgenau School of Engineering, George Mason University, 4400 University Dr, MSN 1J7, Fairfax, VA 22030; e-mail: shadad@gmu.edu

- Indicates open access to non-subscribers at www.ajnr.org

Indicates article with online supplemental data.

http://dx.doi.org/10.3174/ajnr.A7263 circulation, and numerous aneurysms remain patent for a long time after treatment; approximately $25 \%$ remain incompletely occluded after 6 months. ${ }^{3,4}$ Thus, understanding and identifying which aneurysms are likely to quickly occlude and which ones are likely to remain incompletely occluded is important for personalized management-planning and to avoid or minimize retreatment of these aneurysms.

Computational fluid dynamics (CFD) has been previously proposed in several studies as a predictive tool for flow-diversion treatment of intracranial aneurysms. ${ }^{5-10}$ Similarly, alteration of the mean aneurysm flow amplitude, estimated from highframe-rate dynamic DSA, has also been proposed as an outcome predictor for FD treatment. ${ }^{11}$ Therefore, the objective of this study was to evaluate the predictive power of previously reported subject-specific CFD models ${ }^{12}$ by performing a prospective analysis of aneurysm occlusion after FD treatment using elastase rabbit models. 


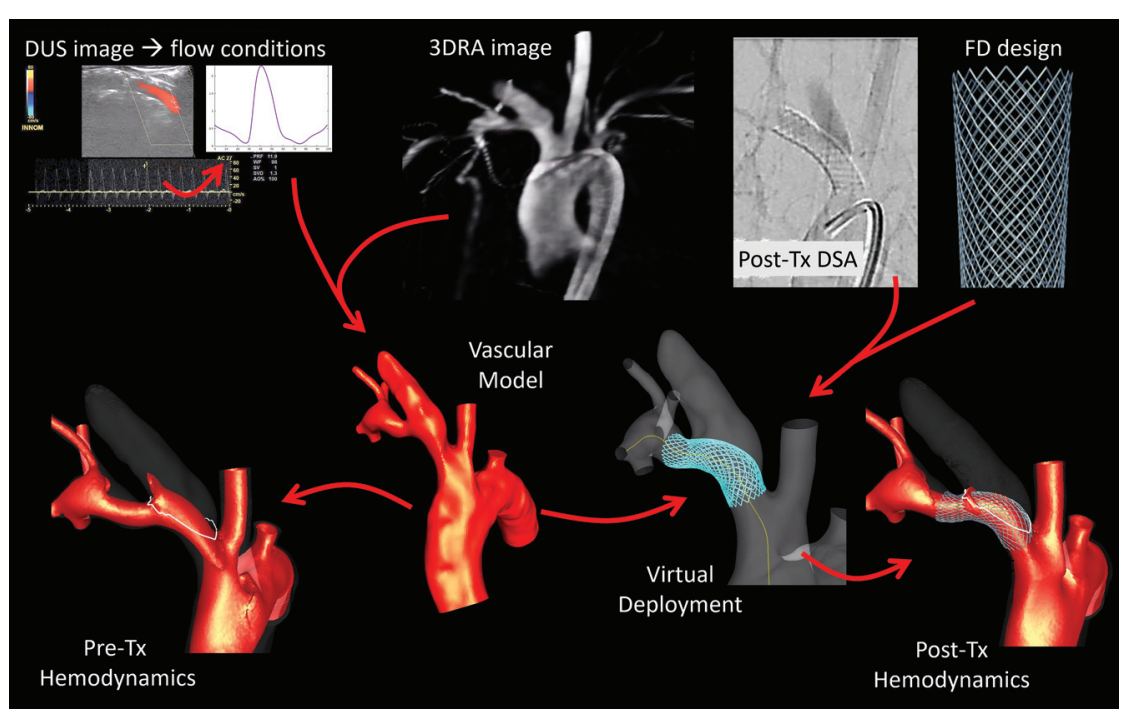

FIG 1. Subject-specific, multimodal image-based CFD modeling methodology. A subject-specific vascular model constructed from a 3D rotational angiography (3DRA) image, flow conditions derived from Doppler ultrasound images, and an FD model constructed from device-design data and virtually deployed into vascular model guided by 2D-DSA. Pre- and posttreatment hemodynamics characterized by corresponding CFD simulations and inflow jets visualized with velocity isosurfaces. Tx indicates Treatment; Pre-Tx, Pretreatment; Post-Tx, Posttreatment.

area to the power $3 / 2$ (the Murray law) were applied. Vascular walls were approximated as rigid, and no-slip boundary conditions were applied at the walls. Simulations were performed for 2 cardiac cycles, and flow fields from the second cycle were used to characterize the aneurysm hemodynamic environment.

\section{FD Modeling}

As in previous studies, ${ }^{6,14}$ models of the flow-diverting device were created and virtually deployed within the vascular models (Fig 1). For this purpose, the skeleton of the parent artery was first reconstructed from the vascular model, and a cylindrical structure was expanded within the vascular model until it contacted the wall or reached the device diameter. The device design (48 wires of $32-\mu \mathrm{m}$ thickness braided into 24 cells with a braid angle of $150^{\circ}$ ) was then mapped onto the deployed cylindrical surface, taking into account the

\section{MATERIALS AND METHODS}

\section{Animal Models and Imaging Data}

A total of 13 aneurysms were experimentally created in rabbits using elastase and carotid ligation techniques previously developed and used to evaluate flow diverters. ${ }^{13}$ The aneurysms were allowed to progress for 4 weeks and then were treated by implantation of a flow-diverting device, the Pipeline Embolization Device (PED; Medtronic). Vessel diameter at the aneurysm neck and device sizes are given in the Online Supplemental Data. A 3D rotational angiography image was acquired immediately before the implantation of the flow diverter, and 2D-DSA sequences were acquired both immediately prior to and immediately after implantation. Doppler ultrasound was used to measure the pretreatment blood flow velocity in the parent artery proximal and distal to the aneurysm. Aneurysm occlusion was subsequently evaluated using DSA sequences acquired 8 weeks after the implantation of the flow-diverting device.

\section{Hemodynamics Modeling}

Subject-specific CFD models were created from the 3D rotational angiography images using a previously described methodology, ${ }^{14}$ illustrated in Fig 1. Briefly, 3D vascular segmentations were used to create anatomic models of the aneurysm and parent artery. Blood flow was mathematically modeled by the unsteady incompressible Navier-Stokes equations, which were numerically solved using a finite element code. ${ }^{15}$ Pulsatile flow conditions were derived from the Doppler ultrasound acquired in the parent artery proximal to the aneurysm. The velocity waveforms were digitized and converted to flow-rate curves by multiplying by the vessel cross-sectional area and were used to prescribe inflow boundary conditions. Outflow boundary conditions consistent with a flow division proportional to the vessel cross-sectional foreshortening effects due to device oversizing. ${ }^{16}$ 2D-DSA images acquired immediately postimplantation (Online Supplemental Data) were used to verify that the position of the virtual FD within the vascular model was consistent with the actual FD depicted in the DSA images. The computational mesh was then adaptively refined to resolve the device wires, and a new flow solution corresponding to the immediate posttreatment conditions was obtained using an immersed boundary method on unstructured grids. ${ }^{17}$

\section{Outcome Prediction and Evaluation}

In a previous study, ${ }^{12}$ a series of 36 aneurysms created in rabbits was used to compare the hemodynamics between aneurysms that were completely occluded at $\leq 4$ weeks (fast-occlusion group) and aneurysms that remained incompletely occluded at 8 weeks (slow- or incomplete-occlusion group). In that study, it was found that the posttreatment mean aneurysm velocity (VEL) and the mean aneurysm inflow rate $(\mathrm{Q})$ were significantly lower in the fast-occlusion group than in the incomplete-occlusion group (VEL, $P=.05 ; \mathrm{Q}, P=.02$ ). Receiver operating characteristic curve analysis suggested that these variables could be used to discriminate between aneurysms in these 2 groups, with a discriminatory power given by the corresponding areas under the receiver operating characteristic curve of 0.83 (VEL) and $0.90(\mathrm{Q})$, respectively. The optimal threshold for each of these 2 variables was determined from the point along the receiver operating characteristic curve closest to the upper-left corner (ie, the threshold that maximizes specificity and sensitivity). The values of these thresholds were $0.161 \mathrm{~cm} / \mathrm{s}$ for VEL, and $0.041 \mathrm{~mL} / \mathrm{s}$ for Q.

Outcome predictions were made for the 13 aneurysms in the current series on the basis of these previously determined thresholds. Specifically, if both the posttreatment VEL and Q were below the corresponding thresholds, the aneurysm was assigned 
Table 1: Posttreatment hemodynamic variables, predictions, outcomes, and agreement

\begin{tabular}{lccccc}
\hline Subject & $\mathbf{Q}(\mathbf{m L} / \mathbf{s})$ & VEL $(\mathbf{c m} / \mathbf{s})$ & Prediction & Outcome & Agreement \\
\hline 1 & 0.055 & 0.391 & $\mathrm{I}$ & $\mathrm{I}$ & $\mathrm{A}$ \\
2 & 0.013 & 0.127 & $\mathrm{C}$ & $\mathrm{I}$ & $\mathrm{D}$ \\
3 & 0.048 & 0.532 & $\mathrm{I}$ & $\mathrm{I}$ & $\mathrm{A}$ \\
4 & 0.013 & 0.112 & $\mathrm{C}$ & $\mathrm{I}$ & $\mathrm{D}$ \\
5 & 0.018 & 0.179 & $\mathrm{I}$ & $\mathrm{I}$ & $\mathrm{A}$ \\
6 & 0.053 & 0.362 & $\mathrm{I}$ & $\mathrm{I}$ & $\mathrm{A}$ \\
7 & 0.031 & 0.344 & $\mathrm{I}$ & $\mathrm{I}$ & $\mathrm{A}$ \\
8 & 0.094 & 0.664 & $\mathrm{I}$ & $\mathrm{I}$ & $\mathrm{A}$ \\
9 & 0.011 & 0.108 & $\mathrm{C}$ & $\mathrm{C}$ & $\mathrm{A}$ \\
10 & 0.007 & 0.156 & $\mathrm{C}$ & $\mathrm{C}$ & $\mathrm{A}$ \\
11 & 0.021 & 0.102 & $\mathrm{C}$ & $\mathrm{C}$ & $\mathrm{A}$ \\
12 & 0.100 & 0.831 & $\mathrm{I}$ & $\mathrm{C}$ & $\mathrm{D}$ \\
13 & 0.025 & 0.156 & $\mathrm{C}$ & $\mathrm{C}$ & $\mathrm{A}$ \\
\hline
\end{tabular}

Note:-I indicates incomplete occlusion; C, complete occlusion; A, agreement; D, disagreement.

Table 2: Summary of agreement between predictions and outcomes

\begin{tabular}{lccc}
\hline & \multicolumn{3}{c}{ Outcomes } \\
\cline { 2 - 4 } Predictions & Complete & Incomplete & Total \\
\hline Complete & 4 & 2 & 6 \\
Incomplete & 1 & 6 & 7 \\
Total & 5 & 8 & 13 \\
\hline
\end{tabular}

to the fast-occlusion group. Alternatively, if either of these 2 variables were above their thresholds, the aneurysm was assigned to the incomplete-occlusion group. All predictions were made prospectively, blinded to the actual outcome.

On the basis of DSA sequences acquired at 8 weeks after treatment with an FD, the outcomes were assessed as complete occlusion (no filling of the aneurysm and no remnant) or incomplete occlusion (persistent filling of the aneurysm or small remnant). Note that because all outcomes were assessed at 8 weeks, a small remnant at 8 weeks would most likely have placed the aneurysm in the slow-occlusion group of the previous study (not in the fastocclusion group in which complete occlusion was required at $\leq 4$ weeks). Finally, the outcomes were compared against the predictions on the basis of the immediate posttreatment CFD analyses.

\section{RESULTS}

The main results of the study are summarized in Table 1. This table lists the values of the hemodynamic variables used to predict outcomes, namely the mean aneurysm $Q$ and mean aneurysm VEL, both corresponding to the hemodynamic conditions immediately after FD implantation. The table also shows, for each aneurysm, the prospective prediction based on both Q and VEL, the actual outcome at 8 weeks, and whether they agreed or disagreed. For completeness, the pre- and posttreatment values of these variable and their relative change are listed in the Online Supplemental Data.

Of the 13 aneurysms treated, 8 (62\%) were angiographically classified as incompletely occluded at 8 weeks, and 5 (38\%), as completely occluded. Sample DSA images acquired immediately before and after treatment and at 8 weeks, follow-up are shown in the Online Supplemental Data for each aneurysm.

A summary of the agreement between predictions and outcomes is presented in Table 2. Of the 13 predictions, 10 agreed with the actual outcomes, corresponding to an overall accuracy of $77 \%$, a sensitivity of $80 \%$, and a specificity of $75 \%$. In 3 cases (23\%), the predictions disagreed with the outcomes. Two of these disagreements corresponded to false-negatives (ie, predicted complete occlusions but aneurysms were incompletely occluded at follow-up), and one, to a false-positive (ie, predicted incomplete occlusion but the aneurysm was completely occluded at follow-up).

Examples of an aneurysm predicted and confirmed to be completely occluded at follow-up and another predicted and confirmed to be incompletely occluded are presented in Fig 2. In the first case, it can be seen that the aneurysm had a strong inflow jet before treatment, but this jet was effectively blocked by the FD device, resulting in low-inflow and low-velocity patterns after treatment. In the second case, the FD device did not sufficiently reduce the inflow and mean velocity, and the aneurysm remained incompletely occluded at follow-up.

\section{DISCUSSION}

Predicting whether an aneurysm will immediately occlude after treatment with an FD device or whether it will remain incompletely occluded is important to plan the best management strategy for each individual patient. For example, it could help support decisions about using multiple devices to achieve the desired hemodynamic environment, choosing a different FD device or endovascular approach, closely monitoring patients who may need retreatment, or determining when to discontinue dual antiplatelet therapy.

The use of image-based CFD models to predict FD outcomes has been proposed in several studies that demonstrated hemodynamic differences between completely and incompletely occluded aneurysms. ${ }^{5-10}$ Furthermore, similar trends have been reported for intrasaccular FDs for the treatment of bifurcation aneurysms that are problematic for endoluminal devices. ${ }^{18}$ The current work focused on validating CFD-based predictions in experimental aneurysms created in rabbits. The study was restricted to endoluminal FDs, and hemodynamic variables previously identified as the principal distinguishing characteristics between fast occlusion and incomplete occlusion after treatment were used to predict outcomes. The same variables were also identified as potential discriminators between fast and incomplete occlusions after FD treatment of human aneurysms, with similar areas under the curve. ${ }^{19}$ Therefore, it is reasonable to expect that the results of the current study will generalize to human aneurysms and be consistent with recent reports relating posttreatment flow velocity and FD outcome in humans. ${ }^{20}$

Our results indicate that the combination of $\mathrm{Q}$ and mean VEL immediately after FD implantation is indeed capable of predicting outcomes with an accuracy of $77 \%$. Specifically, aneurysms with low Q and low mean VEL after treatment (below their corresponding thresholds) are predicted to occlude completely, while 


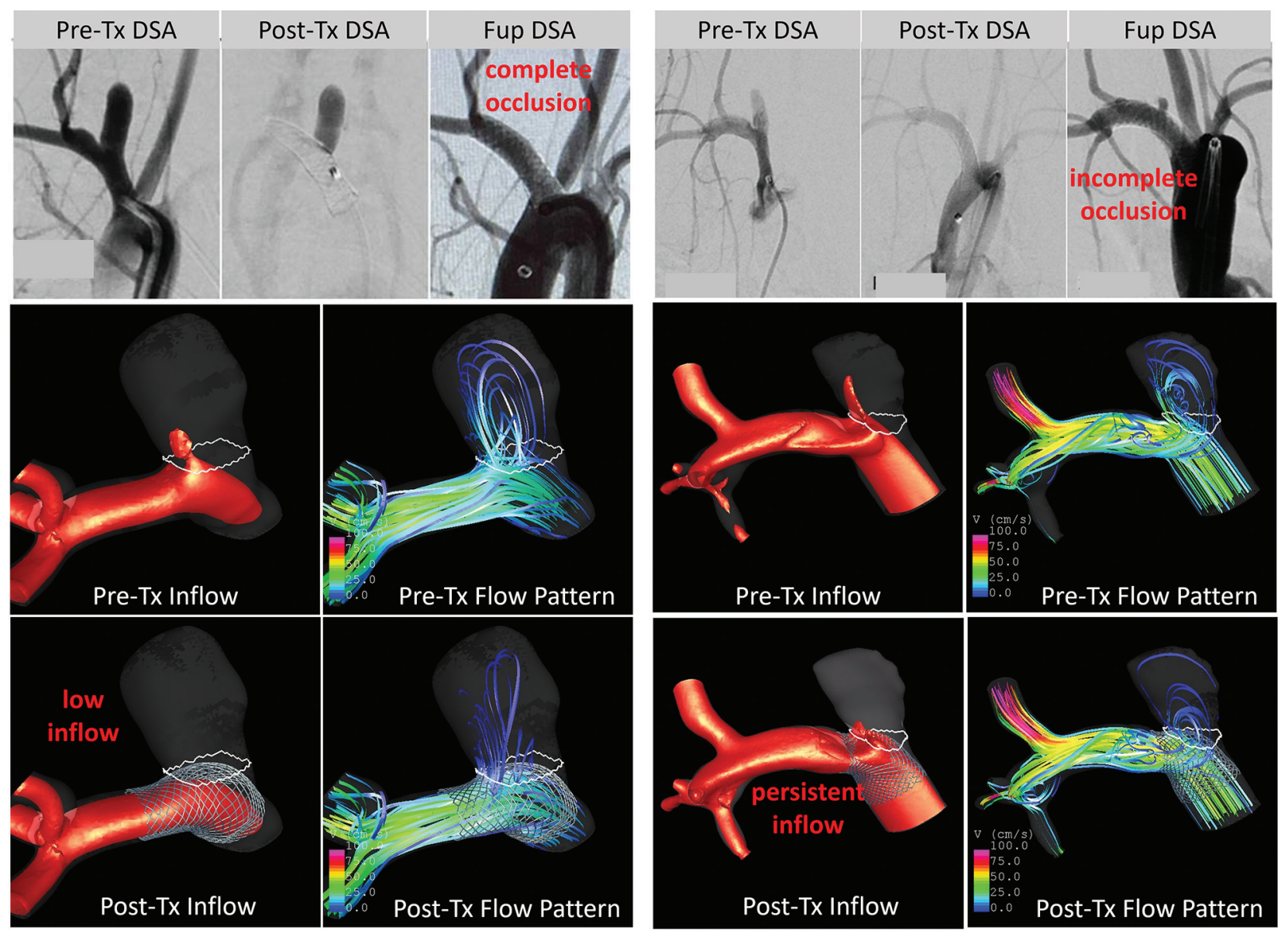

FIG 2. Examples of a completely occluded (left, subject 11) and an incompletely occluded (right, subject 7) aneurysm after treatment with FD devices. In both cases, prospective CFD predictions coincided with the angiographic outcomes at 8 weeks. Visualizations show the inflow jet (isovelocity surfaces) and flow pattern (streamlines) before and after treatment. Substantial reduction of the inflow and flow velocities is observed in the completely occluded aneurysm, but persistent posttreatment inflow and flow velocity can be seen in the incompletely occluded aneurysm. The circled white line show neck area of the aneurysm. Tx indicates Treatment; Fup,follow up; Fup DSA, Follow up DSA.

aneurysms with either a large Q or large mean VEL are predicted to remain incompletely occluded at 8 weeks. Most interesting, if the predictive variables ( $\mathrm{Q}$ and VEL) were considered separately, VEL was able to correctly predict the same $77 \%$ of cases, while Q alone was capable of correctly predicting only $62 \%$ of the cases. This finding suggests that the posttreatment mean aneurysm VEL may be a better predictor than the mean aneurysm inflow Q; however, in the current sample, these 2 variables were not independent (regression analysis showed a linear correlation with an $R^{2}$ value of 0.91 ), explaining why the VEL $+\mathrm{Q}$ combination was not better than the VEL alone. Further studies with more cases are needed to determine whether the combination of these 2 variables can be better than the mean VEL alone in general.

Recently, Paliwal et $\mathrm{al}^{21}$ constructed machine learning models based on 16 morphologic, hemodynamic, and device parameters and applied them to a retrospective cohort of 84 aneurysms treated with FDs and obtained a 90\% accuracy in an internal 20fold cross-validation. In another study, Sindeev et $\mathrm{al}^{22}$ analyzed the flow changes in 3 aneurysms treated with FDs with known outcomes using CFD and phase-contrast MR imaging and concluded that CFD could be used to predict outcomes. Previously, Pereira et $\mathrm{al}^{11}$ studied a prospective series of 24 patients treated with FDs and calculated the mean aneurysm flow amplitude ratio from dynamic DSA images and concluded that this variable could predict complete or incomplete occlusion at 12 months with an accuracy of $86 \%$, sensitivity of $88 \%$, and specificity of $73 \%$. In comparison, we achieved an accuracy of $77 \%$, sensitivity of $80 \%$, and specificity of $75 \%$ when validating the predictions in a prospective manner blinded to the outcomes and in an external data set of 13 rabbit models.

Some of these previous studies have proposed the change in hemodynamic variables such as the mean aneurysm VEL from pretreatment to posttreatment as potential predictors of outcomes. ${ }^{9,13}$ In our previous study, ${ }^{14}$ changes in hemodynamic variables were slightly different between the complete and incomplete aneurysm occlusion groups; thus, it was not possible to use these changes for outcome prediction (ie, it was not possible to calculate corresponding thresholds to discriminate between the 2 groups). However, it was the posttreatment conditions (not their change from the pretreatment values) that were able to predict the outcomes. Large hemodynamic changes may facilitate fast occlusions, but they may not be sufficient if these changes do not produce hemodynamic conditions with values below the predictive thresholds. 
In our study, 2 aneurysms had low-flow conditions after treatment according to the CFD models but remained incompletely occluded at follow-up. These false-negatives are difficult to understand and may indicate that though important, flow conditions may not be the only determining factor for aneurysm occlusion. Perhaps other biologic mechanisms not included in the current models such as individual responses to antiplatelet therapy, diminished fibrin activity, impaired endothelialization, and so forth may slow down the occlusion process. However, in one of these cases (subject 2), the distal parent artery was larger than the FD diameter (device undersized), and consequently, the device was not well-apposed to the wall. This result was confirmed with optical coherence tomography imaging at follow-up (Online Supplemental Data). In such cases, it is very difficult to predict the exact positioning of the FD in the vascular model, and it is possible that the virtual FD was more effective at disrupting the flow into the aneurysm than the actual device, explaining this false-negative.

One case with high-flow conditions after treatment (values above their thresholds) was completely occluded at follow-up (false-positive). Here, we need to remember that the thresholds were determined from a previous study in which one group of aneurysms was completely occluded at 4 weeks, while the other remained open at 8 weeks. Thus, in the current study, perhaps this false-positive case may have been incompletely occluded at 4 weeks but occluded before 8 weeks. These disagreements may also be due to limitations of the CFD models (see below), which could deviate from the actual in vivo hemodynamics in certain subjects. Nevertheless, our models were able to prospectively predict the outcomes correctly in $77 \%$ of the cases. This predictive power is consistent with those in previous studies based on DSA flow assessments ${ }^{13}$ and with the previously determined areas under the curve. ${ }^{14,21}$

In our previous studies, we have found similar hemodynamic differences between complete and incomplete occlusions after FD treatment in rabbits ${ }^{8,14}$ and in humans, ${ }^{21}$ which, in the current study, were used as outcome predictors. Furthermore, we showed that in humans, individualized flow conditions derived from an empirical law relating the inflow rate and parent artery diameter were sufficient to distinguish between complete and incomplete occlusions, ${ }^{21}$ suggesting that it may not be necessary to measure patient-specific flow rates in humans. Additionally, rabbit elastase models have been shown to appropriately mimic human intracranial aneurysms for studying aneurysm treatment. ${ }^{23}$ Thus, it is to be expected that the proposed approach will also work in human aneurysms. The application of CFD modeling of FDs for easy use in the clinic can be simplified by 2 main aspects: 1) developing integrated vascular modeling tools that can be easily interfaced with angiography systems (to accelerate/simplify the manual part of the modeling process), and 2) performing steady-state simulations, which provide good estimates of the mean velocity and inflow rates (accelerating the automatic part of the process). These simplifications would allow CFD models to be created and run in a few minutes. On the other hand, if the hemodynamic variables identified here as good predictors of aneurysm occlusion could be reliably derived from other techniques, such as the mean aneurysm flow amplitude from cine DSA or 4D phase- contrast MR imaging, it may not be necessary to construct CFD models for all patients. However, these other techniques also have limitations that need to be carefully considered (eg, mean aneurysm flow amplitude is a $2 \mathrm{D}$ technique and may underestimate mean velocities in the aneurysm, where the flow has, in general, a complex 3D structure).

The current study has several limitations. The CFD modeling is based on several approximations such as Newtonian flows, rigid vessel walls, inflow rates derived from Doppler ultrasound measurements of flow velocities, and outflow boundary conditions based on the Murray law. However, previous studies showed that these models were able to reproduce in vivo velocity values at the aneurysm neck measured with Doppler ultrasound and overall flow patterns observed in DSA sequences. ${ }^{16}$ The virtual FD deployment methodology takes into account foreshortening effects and is able to reasonably reproduce the geometry of the implanted device, ${ }^{16}$ but it does not exactly reproduce the FD position and shape, which could be affected by operatordependent maneuvers, which could locally compress or expand the device. The sample size was small for both the "training" set used to determine the predictive thresholds of hemodynamic variables, and the "testing" set used to compare the prospective predictions with actual outcomes. Only the PED device was used in this study; thus, the results are exclusively valid for this device. Only 1 device was implanted in each subject; the effects of multiple devices was not studied. Further studies with larger samples and human aneurysms should be performed to confirm our results and further characterize the predictive thresholds of hemodynamic variables (and perhaps develop multivariate models) and the predictive power of CFD models.

\section{CONCLUSIONS}

Subject-specific CFD models of the hemodynamic conditions created immediately after implantation of FD devices in experimental cerebral aneurysms created in rabbits are capable of prospectively predicting (with a reasonable accuracy) which aneurysms will quickly occlude and which ones will remain incompletely occluded for a longer time.

Disclosures: Sara Hadad_RELATED: Grant: National Institutes of Health, Comments: research grant.* Fernando Mut-RELATED: Grant: National Institutes of Health.* Ram Kadirvel_RELATED: Grant: National Institutes of Health, Comments: grant No. NS076491.* David Kallmes_UNRELATED: Grants/Grants Pending: Medtronic, MicroVention, NeuroSigma, NeuroGami Medical, Comments: preclinical research*; Stock/Stock Options: Superior Medical Experts, Comments: founder/owner. Juan R. Cebral—RELATED: Grant: National Institutes of Health, Comments: research grant*; UNRELATED: Grants/Grants Pending: National Institutes of Health, Philips Healthcare, Comments: research grants*; Other: Travis C. Valentine Memorial Fund. *Money paid to the institution.

\section{REFERENCES}

1. Pierot L, Biondi A. Endovascular techniques for the management of wide-neck intracranial bifurcation aneurysms: a critical review of the literature. J Neuroradiol 2016;43:167-75 CrossRef Medline

2. Lylyk P, Miranda C, Ceratto R, et al. Curative endovascular reconstruction of cerebral aneurysms with the Pipeline Embolization Device: the Buenos Aires experience. Neurosurgery 2009;64:632-42 CrossRef Medline 
3. Brinjikji W, Murad MH, Lanzino G, et al. Endovascular treatment of intracranial aneurysms with flow diverters: a meta-analysis. Stroke 2013;44:442-47 CrossRef Medline

4. Kallmes DF, Brinjikji W, Cekirge S, et al. Safety and efficacy of the Pipeline Embolization Device for treatment of intracranial aneurysms: a pooled analysis of 3 large studies. J Neurosurg 2017;127:77580 CrossRef Medline

5. Kulcsar Z, Augsburger L, Reymond P, et al. Flow diversion treatment: intra-aneurismal blood flow velocity and WSS reduction are parameters to predict aneurysm thrombosis. Acta Neurochir (Wien) 2012;154:1827-34 CrossRef Medline

6. Cebral JR, Mut F, Raschi M, et al. Analysis of hemodynamics and aneurysm occlusion after flow-diverting treatment in rabbit models. AJNR Am J Neuroradiol 2014;35:1567-73 CrossRef Medline

7. Paliwal N, Damiano RJ, Davies JM, et al. Association between hemodynamic modifications and clinical outcome of intracranial aneurysms treated using flow diverters. Proc SPIE Int Soc Opt Eng 2017;10135:101352F CrossRef

8. Ma D, Dargush GF, Natarajan SK, et al. Computer modeling of deployment and mechanical expansion of neurovascular flow diverter in patient-specific intracranial aneurysms. J Biomech 2012;45:2256-63 CrossRef Medline

9. Chong W, Zhang Y, Qian Y, et al. Computational hemodynamics analysis of intracranial aneurysms treated with flow diverters: correlation with clinical outcomes. AJNR Am J Neuroradiol 2014;35:13642 CrossRef Medline

10. Huang Q, Xu J, Cheng J, et al. Hemodynamic changes by flow diverters in rabbit aneurysm models: a computational fluid dynamic study based on micro-computed tomography reconstruction. Stroke 2013;44:1936-41 CrossRef Medline

11. Pereira VM, Bonnefous O, Ouared R, et al. A DSA-based method using contrast-motion estimation for the assessment of the intraaneurysmal flow changes induced by flow-diverter stents. AJNR Am J Neuroradiol 2013;34:808-15 CrossRef Medline

12. Chung B, Mut F, Kadirvel R, et al. Hemodynamic analysis of fast and slow aneurysm occlusions by flow diversion in rabbits. $J$ Neurointerv Surg 2015;7:931-35 CrossRef Medline
13. Ding YH, Dai D, Schroeder D, et al. Experimental testing of the dual-layer Woven EndoBridge device using an elastase-induced aneurysm model in rabbits. Interv Neuroradiol 2016;22:299-303 CrossRef Medline

14. Cebral JR, Mut F, Raschi M, et al. Strategy for analysis of flow diverting devices based on multi-modality image-based modeling. Int J Numer Method Biomed Eng 2014;30:951-68 CrossRef Medline

15. Mut F, Löhner R, Chien A, et al. Computational hemodynamics framework for the analysis of cerebral aneurysms. Int J Numer Method Biomed Eng 2011;27:822-39 CrossRef Medline

16. Mut F, Cebral JR. Effects of flow-diverting device oversizing on hemodynamics alteration in cerebral aneurysms. AJNR Am J Neuroradiol 2012;33:2010-16 CrossRef Medline

17. Appanaboyina S, Mut F, Löhner R, et al. Simulation of intracranial aneurysm stenting: techniques and challenges. Comput Methods Appl Mech Eng 2009;198:3567-82 CrossRef

18. Cebral JR, Chung BJ, Mut F, et al. Analysis of flow dynamics and outcomes of cerebral aneurysms treated with intrasaccular flowdiverting devices. AJNR Am J Neuroradiol 2019;40:1511-16 CrossRef Medline

19. Mut F, Raschi M, Scrivano E, et al. Association between hemodynamic conditions and occlusion times after flow diversion in cerebral aneurysms. J Neurointerv Surg 2015;7:286-90 CrossRef Medline

20. Beppu M, Tsuji M, Ishida F, et al. Computational fluid dynamics using a porous media setting predicts outcome after flow-diverter treatment. AJNR Am J Neuroradiol 2020;41:2107-13 CrossRef Medline

21. Paliwal N, Jaiswal P, Tutino VM, et al. Outcome prediction of intracranial aneurysm treatment by flow diverters using machine learning. Neurosurg Focus 2018;45:E7 CrossRef Medline

22. Sindeev S, Arnold PG, Frolov S, et al. Phase-contrast MRI versus numerical simulation to quantify hemodynamical changes in cerebral aneurysms after flow diverter treatment. PLoS One 2018;13:e190696 CrossRef Medline

23. Brinjikji W, Ding YH, Kallmes DF, et al. From bench to bedside: utility of the rabbit elastase aneurysm model in preclinical studies of intracranial aneurysm treatment. J Neurointerv Surg 2016;8:52125 CrossRef Medline 